\title{
Editorial
}

\section{RFID for fashion: Advancements in research, technology and implementation}

The fashion industry is an increasingly complex and dynamic field, characterised by the advent of own brands and the globalization of sourcing and supply chain decisions. This context has recently attracted the attention of several researchers in the area of operations and supply chain management. Accordingly, the main criticalities of the fashion industry include short product life cycle, high volatility, and low predictability of demand and high purchasing impulse. Moreover, as in any other manufacturing business, balancing production and demand is a further challenge faced by fashion retailers, exacerbated by the fact that demand is highly variable and influenced by trends, the short selling season, as well as the need to produce and provide various, complex and fashionable textile products.

Radio frequency identification (RFID) technology offers attractive ways to improve supply chain efficiency through greater coordination between marketing and manufacturing. RFID enables real-time tracking of items, safety monitoring and automation of warehouse operations, which leads to increase in sales volumes and improved profitability for suppliers and retailers. Moreover, RFID implementation in a business organization has a significant impact on the entire supply chain efficiency, from suppliers to retailers. RFID also has the potential to help retailers solve several key issues of the fashion industry. Because of the short product life cycle of fashion items, it is vital to ensure that the product is delivered to the store as quickly as possible. Since RFID provides the capability to track and trace items, it could streamline many activities along the supply chain. Fashion retailers often need space to display products of different styles, sizes and colours; consequently, efficiently managing items at retail stores becomes a relevant task. An RFID-based stock management system can help employees identify the needed items for the customer more quickly and accurately, at the same time enabling frequent inventory counts of high value merchandise. Moreover, RFID gives each clothing item a special identifier, so that counterfeiting and the practice of selling products illegally can be prevented or at least reduced. Finally, RFID is a promising technology for EAS in the apparel industry, where thefts are one of the main causes of shrinkage. 
On the basis of the premise above, our aim with this special issue is to provide stateof-the-art findings about the use of RFID in the fashion field, in terms, for instance, of the design of new RFID solutions for fashion or technological developments in that field, of business models for RFID in fashion, and of RFID implementations in that industry. This special issue consists of 4 papers, with different topics addressed. In the first paper, Hinkka et al. propose a typology of RFID-based tracking solutions designed to fit differing supply chains. The typology developed is based on a case study, involving a logistics service provider interested in designing a tracking solution for different customers in fashion logistics, and four fashion retailers. The proposed typology enables fashion companies to consider which configuration of RFID tracking best fits the requirements and constraints imposed by their supply chain. Moreover, the typology is expected to enable incremental implementation of supply chain-wide tracking. The second paper, by Bevilacqua et al., proposes the implementation of the RFID technology in an Italian company leader in the furniture industry and involved in the fashion field. The analysis grounds on the business process reengineering approach and highlights the benefits of implementing the RFID technology across the company's various business processes. A cost analysis of the reengineered scenarios is also proposed. Rinaldi and Bandinelli, in the third paper, present a practical tool to support practitioners, consultants and researchers in achieving improvements in the effectiveness of logistics processes in the fashion supply chain, by determining the expected profitability of the RFID technology investments. The aim of their approach is to support practitioners and managers in evaluating a priori the profitability of an RFID investment in the fashion supply chain, estimating the related pay-back period; indirectly, this approach is also expected to increase the success of RFID-based project implementations while reducing the uncertainty in terms of economical results. In the fourth paper, Piramuthu et al. develop a protocol to test the performances of RFID devices adopted in the fashion field. The protocol matches the operational conditions of many supply chain processes, but is expressively tailored to the apparel industry. The authors use the protocol to assess the performance of different RFID devices (e.g., inlays, fixed and handheld RFID readers and printers), thus providing a benchmark for their deployment in the apparel logistics.

Thanks to the variety of topics addressed, we believe that this special issue provides the scientific community with valuable information and knowledge related to RFID deployment in the fashion industry. Obviously, the value-added of a special issue is only as good as the contributions of the manuscripts received, and the quality of the feedback provided by the reviewers. Therefore, we are very grateful to all the authors, who supported this special issue through their contributions, and to the reviewers, who helped us in managing the papers received in a timely manner and provided useful and professional reports about their quality. Finally, we would like to express our gratitude to the Editor-in-Chief of International Journal of RF Technologies: Research and Applications, which gave us the possibility of organizing the special issue and helped us in its successful completion. 


\section{Guest-Editors}

Dr. Eleonora Bottani Department of Industrial Engineering, University of Parma Viale G.P.Usberti 181/A, 43124 Parma, Italy

E-mail: eleonora.bottani@unipr.it.
Dr. Sandeep Goyal

College of Business,

Department of Computer

Information Systems,

University of Louisville,

Louisville, KY 40292, USA

E-mail: Sandeep.Goyal

@louisville.edu
Dr. Andrea Volpi

Department of Industrial

Engineering, University of

Parma Viale G.P.Usberti

181/A, 43124 Parma, Italy

E-mail: andrea.volpi@unipr.it. 